\title{
Management of radix entomolaris on permanent mandibular first molar complicated with ledge: a case report
}

\author{
Sobrina Mohamed Khazin, Musliana Mustaffa*
}

Department of Restorative Dentistry, Kulliyyah of Dentistry, International Islamic University Malaysia, Jalan Sultan Ahmad Shah, Bandar Indera Mahkota, 25200 Kuantan, Pahang, Malaysia

\begin{abstract}
Radix entomolaris is a supernumerary root located distolingually on permanent mandibular molars. It is considered as an anatomical variant and usually curved buccolingually. The presence of radix entomolaris can be endodontically challenging to treat and susceptible to endodontic mishaps such as ledge. This report describes the management of ledge in a radix entomolaris with type- 3 curvature on tooth 46 . Ledge was bypassed using pre-curved K-files \#10, \#15, and \#20 sequentially and preparation was continued using step-back technique. Then, the root canal was obturated using gutta percha and root canal sealer. Diagnostic and clinical challenges in managing radix entomolaris, and prevention and management of ledge were also discussed.
\end{abstract}

Keywords: endodontic mishap, distolingual root, glide path, radix entomolaris, root canal treatment

\section{Introduction}

Permanent mandibular first molar (PMFM) is the first permanent teeth to erupt, and its eruption is frequently overlooked leading to early caries and endodontic intervention is often required. Several morphologic variations of the PMFM have been mentioned in the literature and one of the major variants is the presence of radix entomolaris (RE) (Abella et al., 2012) which is a supernumerary root located lingual to the distal root of PMFM (Calberson et al., 2007).

The complexity of the internal anatomy of RE can complicate non-surgical root canal treatment (NSRCT). RE can have severely
Received:

30 November 2021

Revised:

27 January 2022

Accepted:

8 February 2022

Published Online:

4 March 2022

How to cite this article

Mohamed Khazin, S., \&

Mustaffa, M. Management of

radix entomolaris on

permanent mandibular first

molar complicated with ledge: a

case report. IIUM Journal of

Orofacial and Health

Sciences, 3(1), 147-152.

https://doi.org/10.31436/ijoh

s.v3i1.117

Article DOI:

10.31436/ijohs.v3i1.117

*Corresponding author

Address: Kulliyyah of Dentistry, International Islamic University Malaysia, Jalan Sultan Ahmad Shah, Bandar Indera Mahkota, 25200 Kuantan, Pahang, Malaysia.

Telephone: +6095705500

Email:

muslianamustaffa@iium.edu.my drmusliana@gmail.com curved root canal with small radius of curvature (Abella et al., 2012) hence increases the risk of endodontic mishaps such as ledge which can further complicate the treatment modalities.

Presence of ledge can also influence the outcome of NSRCT; may exclude the possibility of thorough disinfection and obturation especially on the root canal system apical to the ledge (Jafarzadeh and Abbott, 2007b). This in turn will lead to persistent endodontic pathosis and posttreatment disease.

This report presents a case of RE in a Malay patient and is aimed to discuss the diagnostic 
and clinical challenges in managing RE, and prevention and management of ledge.

\section{Case Report}

A 36-year-old Malay gentleman was referred from undergraduate clinic for endodontic management of tooth 46 due to severely curved distal root. The patient was medically fit and healthy and presented with asymptomatic fractured temporary restoration on right PMFM (tooth 46) (Figure 1A). The tooth had delayed response to cold test and electric pulp test. Radiographically, presence of carious lesion reaching the mesial pulp horn, severely short distal root, and curved mesial root with signs of apical periodontitis (Figure 1B). Diagnosis of asymptomatic irreversible pulpitis with asymptomatic apical periodontitis was given.

Non-surgical root canal treatment was commenced under local anaesthesia; inferior alveolar nerve blocked using Mepivacaine hydrochloride (2\% Scandonest) and rubber dam isolation, and visually aided with dental operating microscope OPMI ${ }^{\circledR}$ pico (Carl Zeiss, Inc, Oberkochen, Germany). Temporary restoration and carious lesion were removed, and access cavity revealed three distinct root canal orifices. However, the distal root canal orifice is not located at the centre according to the law of symmetry (Krasner and Rankow, 2004), and map of the chamber floor indicates presence of another distal root canal located lingually. The orifice of distolingual root canal was scouted and located far lingually. Calcification was present that hinders the direct view of the root canal and was removed using ultrasonic scaler Start-X \#3 (Denstply Sirona, Switzerland) and the orifice was enlarged lingually (Figure 1E) to allow access to the root canal.

Working length was determined using electronic apex locator Root ZX mini (J Morita Corp, Japan) and verified with periapical radiograph. Radiographic outlines of the distal root canals showed two separate roots with different curvatures (Figure 1C); a curvature of more than 30-degrees was noted on the disto-lingual root canal (Figure $1 \mathrm{C}-\mathrm{D})$. These findings together with the unusual location of the disto-lingual root canal orifice indicated presence of RE.

Prior to root canal preparation, manual glide path was ensured for all root canals using Kfiles \#8, \#10, and \#15 (Dentsply Sirona, Switzerland) followed by rotary PathFile 013, 016, and 019 (Denstply Sirona, Switzerland). During root canal preparation on RE, ledge was created, noticed due to loss of working length. The ledge was managed by pre-curving the tip of $21 \mathrm{~mm}$ length K-file $\# 10$, the curved tip was inserted towards the wall opposite to the ledge, and the K-file was gently slide to the full working length bypassing the ledge. Sequentially, K-files \#15 and \#20 were used in the same manner. Preparation of RE was continued using stepback technique to ISO \#25. The other root canals were prepared using ProTaper Next (Dentsply Sirona, Switzerland) to size X2. $3 \%$ sodium hypochlorite $(\mathrm{NaOCl})$ (Coltène/Whaledent, Switzerland) was copiously used to irrigate the root canals throughout the procedure. Final irrigation protocol was performed using $\mathrm{NaOCl}, 17 \%$ ethylenediaminetetraacetic acid, and $0.2 \%$ chlorhexidine, activated by EndoActivator (Dentsply Sirona, Switzerland).

The root canals were obturated using gutta percha (GP) and AH-Plus sealer (Denstply Detrey, Germany). Warm vertical compaction technique was utilised for all root canals except for RE, where cold lateral compaction was used instead. The GP cone for the RE was pre-curved and treated with $70 \%$ isopropyl alcohol prior to obturation to improve rigidity of GP and maintain the precurved state to bypass the ledge. GP was cut at $2 \mathrm{~mm}$ below orifice (Figure $1 \mathrm{E}$ ) and sealed with composite resin Smart Dentine Replacement (Dentsply Detrey, Germany), obturation radiograph taken (Figure $1 \mathrm{~F}$ ) and composite core placed. Tooth was restored with porcelain-fused to metal crown (Figure $1 \mathrm{G})$ as definitive restoration.

At 6-month and 1-year review, tooth 46 remained asymptomatic and absence of periapical radiolucency radiographically (Figure 1H). 

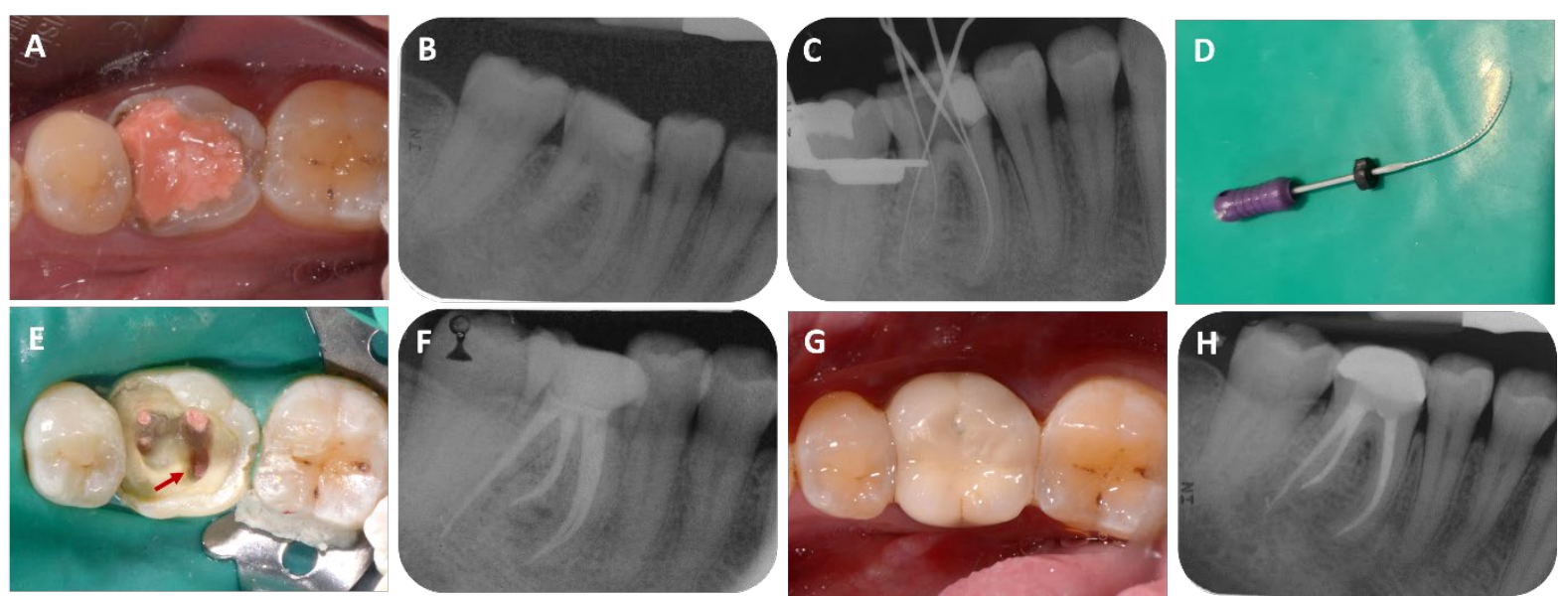

Figure 1. Treatment on tooth 46. A: Pre-operative photograph. B: Pre-operative radiograph. C: Working length radiograph. D: Impression of canal curvature on K-file. E: Clinical photograph of obturation. Orifice of RE is relocated more lingually (arrow). F: Obturation radiograph. G: Cementation of PFM crown. H: 1-year review radiograph

\section{Discussion}

The presence of RE is often overlooked especially due to its rare incidence in Western countries, however, its incidence in Malaysian population can range between $11.3 \%$ to $21.4 \%$ (Deng et al., 2018; Pan et al., 2019). Its presence is significant in endodontics, as it poses several challenges during NSRCT: (1) diagnosis, (2) orifice location, (3) root canal preparation, and (4) root canal obturation.

Diagnosis of RE is important to avoid intraoperative complications or missed canal (Abella et al., 2012), and it can be made through thorough clinical and radiographical examination. The clinical crown can be inspected for the presence of prominent occlusal distal lobe, tuberculum paramolare and cervical prominence (Calberson et al., 2007). However, in this case, minimal coronal tooth structure limits the inspection to diagnose RE.

Periapical radiograph utilizing parallax technique can be used to inspect the root anatomy and prevent overlapping of the distal roots. A mesial view angulated at $25-$ degrees can improve the visibility of $\mathrm{RE}$ compared to distal view (Wang et al., 2011). Three-dimensional radiograph such as cone beam computed tomography (CBCT) can be supplemented to allow clinicians to further visualize and anticipate the complexity of the root anatomy in third dimension whilst eliminating superimposition. The variation of RE can also be further classified according to its morphology and curvature using CBCT (Calberson et al., 2007). The radiation dose for a small field of view CBCT is less than that of conventional CT scan, however based on the concept of "As Low As Reasonably Achievable", CBCT should only be undertaken when more complex anatomy is to be anticipated (Patel et al., 2014). In this case, CBCT was not warranted, but another periapical radiograph should have been taken due to the unclear presence of RE on pre-operative radiograph.

Besides diagnostic challenge, the orifice can be frequently missed due to the presence of calcification. Visual aids such as dental loupes or dental operating microscope can be useful. The law of symmetry and chamber floor map (Krasner and Rankow, 2004) can aid in the location of the orifice. Usually, the orifice will be located more distolingually than the main distal root canal.

The RE is usually curved buccolingually, hence orifice relocation may be warranted to gain straight line access (Figure 1E). However, caution must be taken to prevent strip perforation by avoiding excessive dentine removal due to the slender and curved root. 
RE can have variations in the degree and location of curvature (De Moor et al., 2004). There are three curvature variations: (1) type 1: straight root, (2) type 2: curvature at the cervical third of root and continue as straight root to the apex, and (3) type 3: 2 curvatures; at cervical third and at middle or apical third of the root. It was also reported that most RE has severe curvature of greater than 25-degrees buccolingually.

In this patient's case, a type $3 \mathrm{RE}$ is probable because two curvatures were observed on the initial scouting file (Figure 1D). Having multiple curvatures increases the chance of shaping aberration such as ledge, which occurred in the case reported. Other endodontic mishaps such as perforation, canal transportation and zipping, and instrument separation can also occur.

To manage a curved root canal, it is mandatory for all endodontic hand files to be pre-curved at the tip prior to introducing to the root canal. Small diameter and taper files should be used for initial scouting file such as K-file \#10, to provide critical information regarding the extent and degree of curvature (Alovisi et al., 2016) (Figure1D).

Glide path should also be established prior to shaping. This is to ensure smooth pathway of shaping files to the apex. Previously, the use of rotary nickel-titanium (NiTi) PathFile system was recommended (Abella et al., 2012) however, recently newer rotary glide path files have been introduced with one file system such as ProGlider, Wave One Gold Glider, and TruNatomy Glider. The efficacy of different glide path systems has been investigated, and the results from ex vivo studies utilizing micro-CT have shown that rotary glide path system produces less canal aberrations, canal transportation, and faster working time compared to manual glide path using hand instrument (Pasqualini et al., 2012; Alovisi et al., 2016; Paleker and van der Vyver, 2016; Vorster et al., 2018). Comparable results were also seen between different rotary glide path systems in preserving the root canal anatomy (Plotino et al., 2020). A systematic review and metaanalysis have shown that rotary glide path systems reduce debris extrusion and improves the preservation of root canal anatomy after preparation. However, the effect of glide path on the shaping ability of consequent rotary $\mathrm{NiTi}$ instruments remained unclear (Plotino et al., 2020).

Ledge can be prevented by enlarging the root canal orifice and removing the dentinal shelf to reduce the degree of curvature and gained straight line access coronally. Furthermore, pre-curving the file prior to insertion is also necessary. Additionally, endodontic files should not be forced apically but negotiated slowly apically with adequate lubricant using balanced-force technique. However, in this case, enlarging the orifice further is risky; RE is slender and liable to strip perforation. Pre-curving ProTaper Next is not possible without permanently distorting the file, due to the metallurgy. It is preferable to use martensitic NiTi file such as Hyflex, ProTaper Gold, or TruNatomy in this case.

The first step in managing ledge is to recognise the ledge. There will be a loss of tactile sensation of the tip of the instrument binding into the root canal, and the feeling of instrument hitting a solid wall (Figure 2A). The second step is to bypass the ledge through negotiation with endodontic file. Shortest file that can reach the working length should be used. This will allow better control when manoeuvring the file in the root canal. Introduce a small (\#10 or \#15) pre-curved file tip towards the wall opposite to the ledge (Figure 2B), with slight rotation motion together with "picking" motion to allow advancement of the file, bypassing the ledge, and gently slide it to the full working length. However, if there is resistance, and the file cannot advance, changing the direction of the curved tip and repeating the process can be done until the ledge can be bypassed. The use of $\mathrm{C}+$ file have been introduced to ease in the attempt of bypassing ledge. To remove the ledge, the file should be maintained apical to ledge but coronal to foramen to prevent unnecessary enlargement of apical foramen. Utilising a filing motion and occasionally very short push-pull movements to remove the ledge is effective (Figure 2C). Adequate lubrication and frequent irrigation are needed to 
prevent debris accumulation and canal blockage. Once ledge is removed, root canal preparation can be continued using rotary NiTi instruments (Jafarzadeh and Abbott, 2007a). In this case, although ledge was bypassed, step-back technique using K-files were used for apical preparation.
A

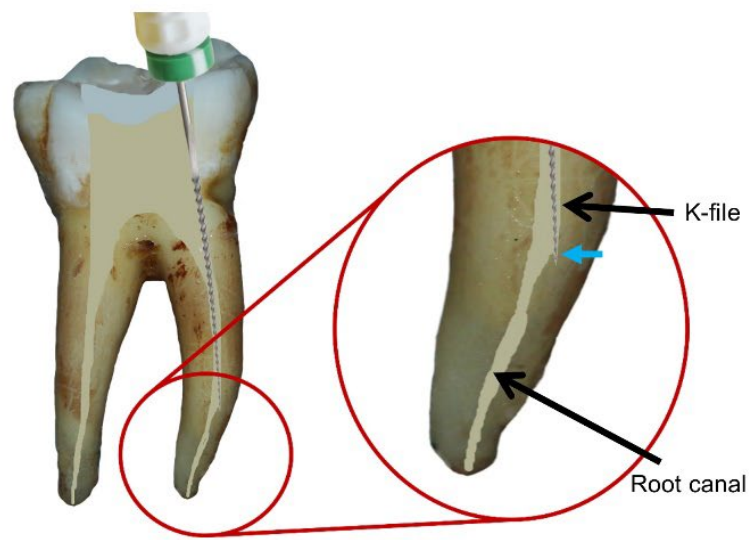

B

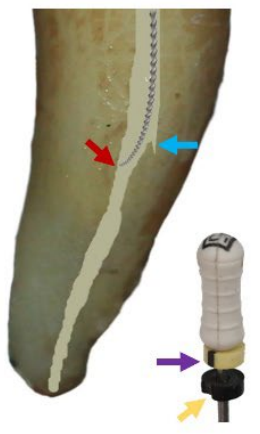

C

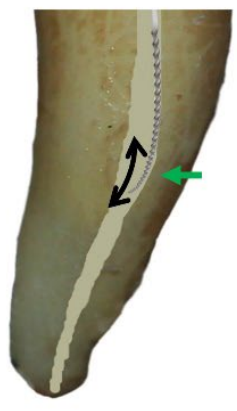

Figure 2. Bypassing and removing ledge. A: Ledge formation (blue arrow). B: To bypass the ledge, pre-curved tip of endodontic file (red arrow) is directed opposite to the ledge (blue arrow) guided by the line (purple arrow) or notch (yellow arrow) on the rubber stopper. C: To remove ledge, brushing the canal wall apical to the ledge (green arrow)

Obturation of a curved root canal can be performed using several techniques; warm vertical compaction, cold lateral compaction, and carrier based obturation (Jafarzadeh and Abbott, 2007b). When cold lateral compaction is opted, NiTi finger spreader is advocated to improve the depth of penetration of spreader into the curved root canal. However, in the case of severely curved root canal, thermoplastic or carried based gutta percha (GP) might be more applicable (Jafarzadeh and Abbott, 2007b).

Obturation of a ledged root canal, however, poses a greater challenge; GP cone might not be able to bypass the ledge and obturation will be short of the working length. This can be improved by pre-curving the GP tip and treated with $70 \%$ isopropyl alcohol to increase the rigidity to bypass the ledge (Jafarzadeh and Abbott, 2007b), which was performed in this case, and was able to be obturated to the working length (Figure 1E). Alternatively, thermoplastic GP using continuous wave technique (Jafarzadeh and Abbott, 2007a) or obturation using bioceramic root canal sealers can be used to be injected into the root canal.

At 1-year review, there were evidence of healed bony lesion at the periapex of tooth 46. Despite the complication of ledge, the RE was able to be cleaned, shaped, and obturated to the working length, which have contributed to the favourable prognosis of the tooth. It is to be suggested that clinicians should be updated on the endodontic instruments and materials to help in the prevention and management of mishaps, and have sound knowledge and understanding on root canal morphology associated with $\mathrm{RE}$ to improve the endodontic treatment outcome.

\section{Acknowledgement}

This research was funded by IIUM-UMPUiTM Sustainable Research Collaboration Grant 2020 (SRCG) from International Islamic University Malaysia (SRCG20-0140014).

\section{References}

Abella, F., Patel, S., Durán-Sindreu, F., Mercadé, M., Roig, M. (2012). Mandibular first molars with disto-lingual roots: review and clinical management', International Endodontic Journal, 45(11), 963-978.

Alovisi, M., Cemenasco, A., Mancini, L., Paolino, D., Scotti, N., Bianchi, C.C., Pasqualini, D. (2017). Micro-CT evaluation of several glide path techniques and ProTaper Next shaping outcomes in maxillary first molar curved 
canals. International Endodontic Journal,50(4), 387-397.

Calberson, F.L., De Moor, R.J., Deroose, C.A. (2007). The radix entomolaris and paramolaris: clinical approach in endodontics. Journal of Endodontics, 33(1), 58-63.

Deng, P.U., Halim, M.S., Masudi, S.A.M., Al-Shehadat, S., Ahmad, B. (2018). Cone-beam computed tomography analysis on root and canal morphology of mandibular first permanent molar among multiracial population in East Coast Malaysian population. European Journal of Dentistry, 12(03), 410-416.

Jafarzadeh, H., Abbott, P.V. (2007a). Dilaceration: review of an endodontic challenge.Journal of Endodontics, 33(9), 1025-1030.

Jafarzadeh, H., Abbott, P.V. (2007b). Ledge formation: review of a great challenge in endodontics. Journal of endodontics, 33(10), 1155-1162.

Krasner, P., Rankow, H.J., 2004. Anatomy of the pulpchamber floor. Journal of Endodontics, 30(1), 5-16.

De Moor, R.J.G., Deroose, C.A.J.G., Calberson, F.L.G. (2004). The radix entomolaris in mandibular first molars: an endodontic challenge. International Endodontic Journal, 37(11), 789-799.

Paleker, F., van der Vyver, P.J. (2016). Comparison of canal transportation and centering ability of $\mathrm{K}$ files, ProGlider File, and G-Files: a micro-computed tomography study of curved root canals. Journal of Endodontics, 42(7), 1105-1109.

Pan, J.Y.Y., Parolia, A., Chuah, S.R., Bhatia, S., Mutalik, S., Pau, A. (2019). Root canal morphology of permanent teeth in a Malaysian subpopulation using cone-beam computed tomography. BMC Oral Health, 19(1), 1-15.

Pasqualini, D., Bianchi, C.C., Paolino, D.S., Mancini, L., Cemenasco, A., Cantatore, G., et al. (2012). Computed micro-tomographic evaluation of glide path with nickel-titanium rotary PathFile in maxillary first molars curved canals. Journal of Endodontics, 38(3), 389-393.

Patel, S., Durack, C., Abella, F., Roig, M., Shemesh, H., Lambrechts, P., et al. (2014). European Society of Endodontology position statement: the use of CBCT in endodontics. International Endodontic Journal, 47(6), 502-504.

Plotino, G., Nagendrababu, V., Bukiet, F., Grande, N.M., Veettil, S.K., De-Deus, G., et al. (2020). Influence of negotiation, glide path, and preflaring procedures on root canal shaping-terminology, basic concepts, and a systematic review. Journal of Endodontics, 46(6), 707-729.

Vorster, M., van der Vyver, P.J., Paleker, F. (2018). Canal transportation and centering ability of WaveOne Gold in combination with and without different glide path techniques. Journal of Endodontics, 44(9), 1430-1435.

Wang, Q., Yu, G., Zhou, X.D., Peters, O.A., Zheng, Q.H., Huang, D.M. (2011). Evaluation of X-ray projection angulation for successful radix entomolaris diagnosis in mandibular first molars in vitro. Journal of Endodontics, 37(8), 1063-1068. 